\title{
Factors associated with prolonged overall survival in patients with postmenopausal estrogen receptor-positive advanced breast cancer using real-world data: a follow-up analysis of the JBCRG-C06 Safari study
}

\author{
Hidetoshi Kawaguchi ${ }^{1}\left(\right.$ Norikazu Masuda $^{2} \cdot$ Takahiro Nakayama $^{3} \cdot$ Kenjiro Aogi $^{4} \cdot$ Keisei Anan $^{5} \cdot$ Yoshinori Ito $^{6}$. \\ Shoichiro Ohtani ${ }^{7} \cdot$ Nobuaki Sato $^{8} \cdot$ Shigehira Saji $^{9} \cdot$ Toshimi Takano $^{10} \cdot$ Eriko Tokunaga $^{11}$. Seigo Nakamura ${ }^{12}$. \\ Yoshie Hasegawa ${ }^{13} \cdot$ Masaya Hattori $^{14} \cdot$ Tomomi Fujisawa $^{15}$. Satoshi Morita ${ }^{16} \cdot$ Miki Yamaguchi $^{17}$. \\ Hiroko Yamashita ${ }^{18}$. Toshinari Yamashita $^{19}$. Yutaka Yamamoto ${ }^{20}$. Daisuke Yotsumoto ${ }^{21} \cdot$ Masakazu Toi $^{22}$. \\ Shinji Ohno ${ }^{23}$
}

Received: 24 June 2019 / Accepted: 28 November 2019 / Published online: 6 December 2019

(c) The Author(s) 2019

\begin{abstract}
Background Assessing survival risk is important for discussing treatment options with estrogen receptor-positive (ER+) advanced breast cancer $(\mathrm{ABC})$ patients. However, there are few reports from large-scale databases on the survival risk factors in ER+ ABC. The Safari study (UMIN000015168) was a retrospective, multicenter cohort study involving 1072 Japanese patients receiving fulvestrant $500 \mathrm{mg}$ mostly as a second- or later-line endocrine therapy for ER+ ABC. The follow-up data after the Safari study were examined, focusing on any relationship between clinicopathological factors and overall survival $(\mathrm{OS})$ in $\mathrm{ER}+\mathrm{ABC}$ patients.

Methods OS in patients with $\mathrm{ER}+\mathrm{ABC}$ was analyzed by univariate and multivariate analyses with a Cox proportional hazards model in this study.

Results A total of 1031 cases were evaluable for OS analysis. Multivariate analysis showed that younger age ( $<60$ years), longer time from $\mathrm{ABC}$ diagnosis to fulvestrant use ( $\geq 3$ years), no prior palliative chemotherapy before fulvestrant use, and progesterone receptor $(\mathrm{PgR})$ negativity $(\mathrm{PgR}-)$ were significantly correlated with prolonged OS (median 7.0 years). For cases with histological or nuclear grade data, lower histological or nuclear grades were also correlated with longer OS. In recurrent metastatic cases, long disease-free interval (DFI) was not correlated with longer OS.

Conclusions In $\mathrm{ER}+\mathrm{ABC}$ patients whose treatment history included fulvestrant, younger age, longer time from $\mathrm{ABC}$ diagnosis to fulvestrant use, no prior palliative chemotherapy use, $\mathrm{PgR}-$, and lower histological or nuclear grade correlated positively with prolonged OS.
\end{abstract}

Keywords Breast cancer $\cdot$ Antineoplastic agents $\cdot$ Post-menopause $\cdot$ Hormones $\cdot$ Overall survival

Electronic supplementary material The online version of this article (https://doi.org/10.1007/s12282-019-01029-3) contains supplementary material, which is available to authorized users.

Hidetoshi Kawaguchi

hkawaguchi@matsuyama.jrc.or.jp

Extended author information available on the last page of the article

\section{Introduction}

Most breast cancer patients who are diagnosed at an early stage have a good clinical outcome [1]. However, nearly $30 \%$ of patients newly diagnosed with early-stage breast cancer will later develop recurrent metastatic cancer [2], for whom the 5 -year survival rate is approximately $20 \%$ [3]. In advanced breast cancer (ABC) (including locally advanced and metastatic breast cancer [4]) patients, the disease currently remains incurable [5]. In ABC, estrogen receptorpositive $(\mathrm{ER}+)$ is the most common subtype [6]. 
Recently, several drugs, such as selective estrogen receptor modulators (SERMs), selective estrogen receptor downregulators (SERD), aromatase inhibitors (AIs), cyclin dependent kinase (CDK) 4/6 inhibitors, and mTOR inhibitor have been used to treat $\mathrm{ER}+\mathrm{ABC}$. Other chemotherapy regimens as well as bevacizumab are also effective, especially for high-risk cases [7] or cases suspected to be endocrine therapy resistant. Selecting drugs to maximize overall survival (OS) and quality of life for $\mathrm{ER}+\mathrm{ABC}$ is obviously desirable. Unfortunately, the tumor burden and tumor biology differ between initial diagnosis and recurrence, and patients may have become endocrine therapy resistant because they continued endocrine therapy as adjuvant treatment $[8,9]$. However, there are few reports from large-scale data sets on the prognostic factors for $\mathrm{ABC}$ [10-12], especially focusing on $\mathrm{ER}+\mathrm{ABC}[6,13,14]$.

We constructed a database of more than $1000 \mathrm{ER}+$ breast cancer cases in the Safari study. The Safari study examined the association between clinicopathological factors and time to treatment failure (TTF) of fulvestrant in Japanese ABC patients who received fulvestrant $[15,16]$. Fulvestrant is a selective estrogen receptor degrader that is administered to patients with ER+ ABC [17, 18]. We continued to collect prognostic data after the main analysis of the Safari study was completed.

The objective of this OS analysis was to examine the data from the Safari study with a focus on the potential effect that patient- or disease-related factors [19] may have on OS.

\section{Materials and methods}

\section{OS analysis design}

The study design and patient cohort for the Safari study (UMIN000015168) have been published previously [15, 16]. In brief, Safari study was a retrospective, multicenter cohort study that examined the association between clinicopathological factors and TTF of fulvestrant in Japanese ABC (JBCRG-C06 Safari) $[15,16]$. The study was conducted in accordance with the Helsinki Declaration, "Guidelines for Clinical Evaluation Methods of Anti-Cancer Drugs" and "Ethical Guidelines for Epidemiology Research (revised on December 1, 2008)". The protocol was approved at each institute. Patients were followed according to the guidelines of the Japan Breast Cancer Society and the National Comprehensive Cancer Network (NCCN) Guidelines for Invasive Breast Cancer (Version 4.2018) [20], which is the standard treatment in Japan. This study was registered as UMIN000015168.

Patients starting fulvestrant treatment between 25 November 2011 (fulvestrant approval date in Japan) and 31 December 2014 were registered. The data cut-off for primary analysis was 27 September 2015 and for OS analysis was 30 April 2018.

We analyzed OS using the same factors of TTF as previously reported $[15,16]$ because we wanted to compare the results of this study with those of our previous report regarding TTF. Factors investigated were patient age ( $\geq 60$ years vs. $<60$ years), fulvestrant treatment line ( $\geq 4$ th vs. 3 rd vs. 1 st and 2nd), period from $\mathrm{ABC}$ diagnosis to fulvestrant use ( $\geq 3$ years vs. $<3$ years), histological or nuclear grade ( 2 vs. 1,3 vs. 1 ), visceral metastasis (yes vs. no), progesterone receptor (PgR) expression (positive vs. negative), human epidermal growth factor receptor 2 (HER2) expression (positive vs. negative), disease-free interval (DFI) ( $\geq 5$ vs. $<5$ years), and prior palliative chemotherapy use (yes vs. no). "Prior palliative chemotherapy use" means any chemotherapy received after the diagnosis of $\mathrm{ABC}$ to the start of fulvestrant, and excludes any neoadjuvant or adjuvant chemotherapy.

In this analysis, all cut-off other than age and DFI were matched to the main analysis of TTF. The median age of 65 years was the cut-off in the TTF analysis, and the median age of 60 years was the cut-off in the OS analysis. The reason why the median age differed between the TTF analysis and the OS analysis is because the starting point of each analysis was different. The starting point for the TTF analysis was the start date of the fulvestrant treatment, while the starting point of the OS analysis was the start of treatment for advanced cancer, and the median age at that time was 5 years younger than the start of treatment for fulvestrant. Regarding the DFI cut-off, in our previous report we used "DFI ( $\geq 2$ vs. $<2$ years)". We used a cut-off of 2 years, as explained in the ESO-ESMO guidelines about intrinsic or primary endocrine resistance for early breast cancer, if defined as recurrence within the first 2 years of adjuvant endocrine therapy [4]. Nonetheless, in this analysis, we wanted to examine factors related to OS rather than endocrine therapy sensitivity, so the cut-off was set at a median of 5 years instead of 2 years.

\section{Statistical methods}

OS was defined as the duration from the date of initial treatment of recurrent breast cancer to death. Kaplan-Meier curves were also used for analysis to investigate OS. Subjects whose deaths were not documented by the time of data cut-off were censored when survival was last documented. The Cox hazards model was used to evaluate the relationship between each of the clinical factors and OS. We also performed multivariate analysis on OS using factors that had a statistical difference $(P<0.1)$ in univariate analysis. Hazard ratios (HRs) with $95 \%$ confidence intervals (CIs) and $P$ values are described. All tests were two-sided and $P<0.05$ was considered statistically significant. 


\section{Results}

\section{Patient demographics}

A total of 1072 cases from 16 centers were enrolled in the Safari study. A CONSORT diagram is shown in Fig. 1. Thirty-seven cases were not eligible because they combined fulvestrant with other therapies (such as endocrine therapy, and/or chemotherapy, and/or target therapy). However, HER2-combined cases were allowed. Four cases were also excluded from the analysis because they were estrogen receptor-negative (ER-). Finally, 1031 (96.2\%) patients with $\mathrm{ER}+\mathrm{ABC}$ were eligible for the OS analysis. After the main analysis of TTF, we collected follow-up data for treatment and OS. When we collected these data, we queried PgR and HER2 status because there were some unknown or unclear cases. For the receptor status determination, data that could be determined before the administration of fulvestrant were used in the analysis of TTF, but in this study, data that could be determined when $A B C$ was diagnosed were used. As a result, PgR and HER2 status were changed as follows: $\mathrm{PgR}$ positivity went from 805 to 765 cases, negativity from 187 to 198 cases, and unknown from 39 to 68 cases, while HER2 positivity went from 117 to 94 cases, negativity from 819 to 884 cases, and unknown from 95 to 53 cases (Supplementary Table 1).

Table 1 shows the baseline characteristics of OS data sets. The median age was 60.0 years, visceral metastasis was found in $408(57.5 \%)$ cases, and central nervous metastasis was observed in eight (1.4\%) cases. Most cases were $\mathrm{ER}+\mathrm{PgR}+$, and fulvestrant was used most often for late-line treatment (4th line or later).

\section{Analysis outcomes}

\section{Analysis of all cases}

Median OS was 7.0 years (95\% CI 6.6-7.5 years) (Fig. 2). By univariate analysis (Table 2$)$, younger age ( $<60$ years), earlier treatment line of fulvestrant, longer time from $\mathrm{ABC}$ diagnosis to fulvestrant use ( $\geq 3$ years), no prior palliative chemotherapy use, lower histological or nuclear grade, and $\mathrm{PgR}$ negativity $(\mathrm{PgR}-)$ were associated with longer OS $(P<0.1)$. In the treatment line of fulvestrant, visceral metastases and HER2 status had no effect on OS (Table 2).

By multivariate analysis, younger age $(<60$ years; $P<0.001$ ), longer time from ABC diagnosis to fulvestrant use ( $\geq 3$ years; $P<0.001$ ), no prior palliative chemotherapy use $(P<0.001)$, and PgR- $(P=0.013)$ were correlated with prolonged OS (Table 2). Histological or nuclear grade were not included in this multivariate analysis because data were missing in one-third of cases.

\section{Analysis of all cases with available histological or nuclear grade data}

A subgroup analysis was performed on 679 cases that had histological or nuclear grade data. The patients used in this analysis decreased from 691 cases of TTF to 679 cases. The reason was that the TTF analysis was targeted to those whose histological or nuclear grade could be determined before administration of fulvestrant, but in this study, it was limited to cases that could be determined at the time of diagnosis of $\mathrm{ABC}$. In other words, grade data includes data from the first diagnosis of breast cancer and metastatic site data at the time of recurrence. However, data obtained during palliative treatment from the diagnosis of $\mathrm{ABC}$ has not been
Fig. 1 Patient flow diagram. $E R$ estrogen receptor, HER2 human epidermal growth factor receptor 2
CONSORT diagram

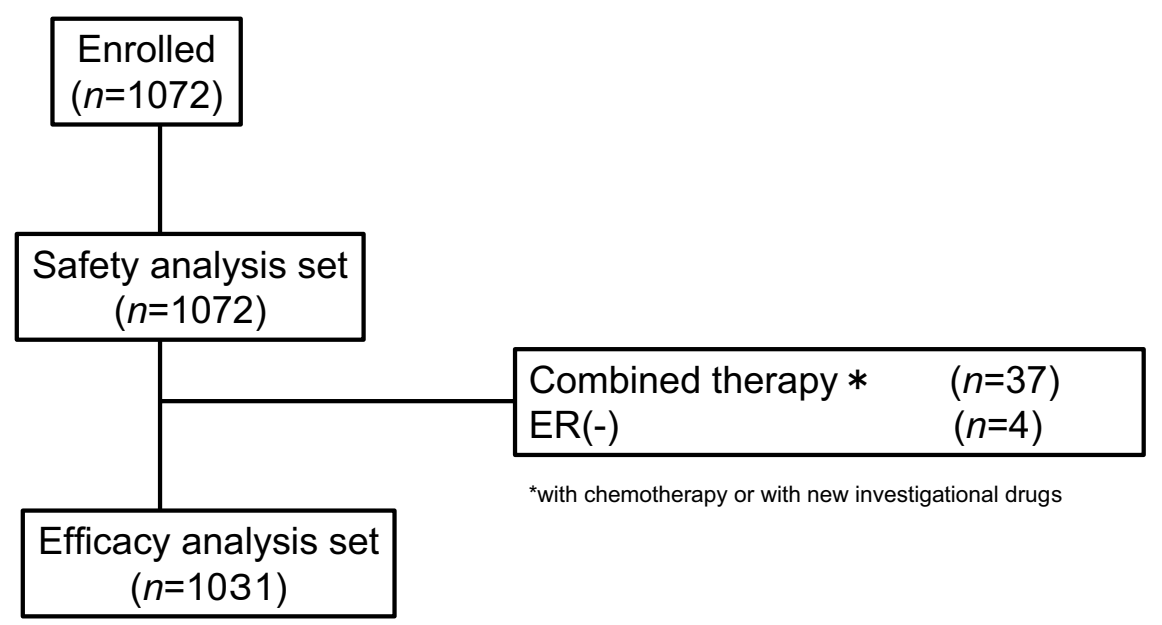


Table 1 Summary of patient characteristics

\begin{tabular}{|c|c|c|c|}
\hline & Efficacy analysis sets & $\begin{array}{l}\text { De novo metastatic or } \\
\text { locally advanced }\end{array}$ & Recurrent metastatic \\
\hline Characteristic & $n=1031$ & $n=207$ & $n=824$ \\
\hline \multicolumn{4}{|c|}{ Age (ABC diagnosis), years } \\
\hline Median & 60.0 & 60.0 & 60.0 \\
\hline Range & $29-91$ & $35-90$ & 29-91 \\
\hline \multicolumn{4}{|c|}{ Age group (ABC diagnosis), $(n[\%])$} \\
\hline$<60$ & $486(47.1)$ & $97(46.9)$ & $389(47.2)$ \\
\hline$\geq 60$ & $545(52.9)$ & $110(53.1)$ & $435(52.8)$ \\
\hline \multicolumn{4}{|c|}{ Fulvestrant treatment lines } \\
\hline $1 \mathrm{st}$ & $21(2.0)$ & $3(1.4)$ & $18(2.2)$ \\
\hline 2nd & $232(22.5)$ & $50(24.2)$ & $182(22.1)$ \\
\hline 3 rd & $276(26.8)$ & $59(28.5)$ & $217(26.3)$ \\
\hline 4th or more & $502(48.7)$ & $95(45.9)$ & $407(49.4)$ \\
\hline \multicolumn{4}{|c|}{$\mathrm{ABC}$ diagnosis to fulvestrant use, years } \\
\hline Median & 3.4 & 3.2 & 3.4 \\
\hline Range & $0-26.9^{\mathrm{a}}$ & $0-18.2$ & $0-26.9^{\mathrm{b}}$ \\
\hline \multicolumn{4}{|c|}{$\mathrm{ABC}$ diagnosis to fulvestrant use, group, years } \\
\hline$<3$ & $473(45.9)$ & $96(46.4)$ & $377(45.8)$ \\
\hline$\geq 3$ & $557(54.0)$ & $111(53.6)$ & $446(54.1)$ \\
\hline Missing & $1(0.1)$ & 0 & $1(0.1)$ \\
\hline \multicolumn{4}{|l|}{ DFI, years } \\
\hline Median & NA & NA & 5.5 \\
\hline Range & NA & NA & $0-31.8^{\mathrm{b}}$ \\
\hline \multicolumn{4}{|l|}{ DFI, group, years } \\
\hline$<5$ & NA & NA & $380(46.1)$ \\
\hline$\geq 5$ & NA & NA & $443(53.8)$ \\
\hline Missing & NA & NA & $1(0.1)$ \\
\hline \multicolumn{4}{|l|}{ Visceral metastasis } \\
\hline No & $588(57.0)$ & $106(51.2)$ & $482(58.5)$ \\
\hline Yes & $443(43.0)$ & $101(48.8)$ & $342(41.5)$ \\
\hline \multicolumn{4}{|c|}{ Central nerve metastasis } \\
\hline No & 1017 (98.6) & $203(98.1)$ & $814(98.8)$ \\
\hline Yes & $14(1.4)$ & $4(1.9)$ & $10(1.2)$ \\
\hline \multicolumn{4}{|l|}{ Histological type } \\
\hline IDC & $863(83.7)$ & $173(83.6)$ & $690(83.7)$ \\
\hline ILC & $48(4.7)$ & $7(3.4)$ & $41(5.0)$ \\
\hline Other & $120(11.6)$ & $27(13.0)$ & $93(11.3)$ \\
\hline \multicolumn{4}{|c|}{ Histological or nuclear grade } \\
\hline 1 & $314(30.5)$ & $48(23.2)$ & $266(32.3)$ \\
\hline 2 & $240(23.3)$ & $54(26.1)$ & $186(22.6)$ \\
\hline 3 & $125(12.1)$ & $28(13.5)$ & $97(11.8)$ \\
\hline NA & $352(34.1)$ & $77(37.2)$ & $275(33.4)$ \\
\hline \multicolumn{4}{|l|}{ Hormonal receptor } \\
\hline $\mathrm{ER}(+) \operatorname{PgR}(-)$ & $198(19.2)$ & $36(17.4)$ & $162(19.7)$ \\
\hline $\mathrm{ER}(+) \operatorname{PgR}(+)$ & $765(74.2)$ & $159(76.8)$ & $606(73.5)$ \\
\hline ER(+) PgR(NA) & $68(6.6)$ & $12(5.8)$ & $56(6.8)$ \\
\hline \multicolumn{4}{|l|}{ HER2 } \\
\hline Negative & $884(85.7)$ & $174(84.1)$ & $710(86.2)$ \\
\hline Positive & $94(9.1)$ & $21(10.1)$ & $73(8.9)$ \\
\hline Missing & $53(5.1)$ & $12(5.8)$ & $41(5.0)$ \\
\hline
\end{tabular}


Table 1 (continued)

\begin{tabular}{llll}
\hline & Efficacy analysis sets & $\begin{array}{l}\text { De novo metastatic or } \\
\text { locally advanced }\end{array}$ & Recurrent metastatic \\
\hline \multicolumn{2}{l}{$\begin{array}{lll}\text { Prior palliative chemotherapy use } \\
\text { No }\end{array}$} & & \\
Yes & $548(53.2)$ & $95(45.9)$ & $453(55.0)$ \\
\hline
\end{tabular}

Results are $n(\%)$ unless otherwise noted

$A B C$ advanced breast cancer, $D F I$ disease-free interval, $E R$ estrogen receptor, HER2 human epidermal growth factor receptor 2, IDC invasive ductal carcinoma, $I L C$ invasive lobular carcinoma, $P g R$ progesterone receptor

${ }^{\mathrm{a}} n=1030$

${ }^{\mathrm{b}} n=823$

OS: All patients $(n=1,031)$

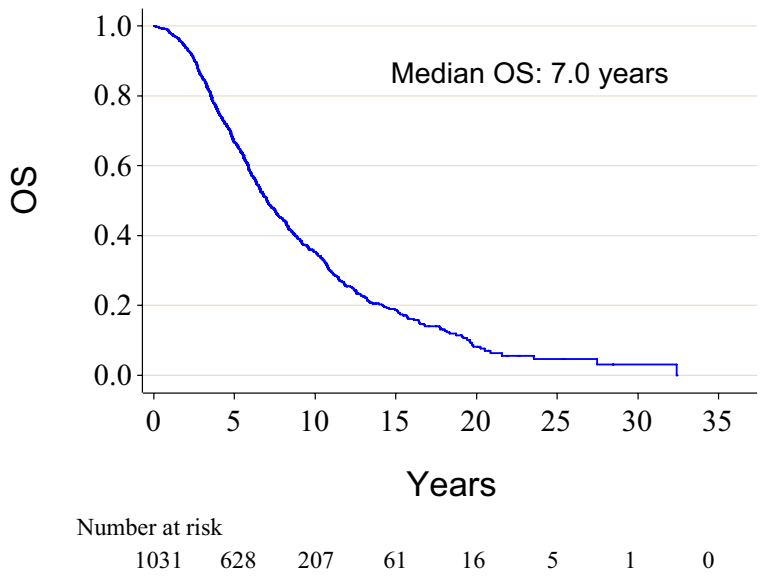

Fig. 2 Kaplan-Meier estimates for OS in all cases. OS overall survival included, even from a biopsy of a local recurrence site or a metastatic recurrence site. Multivariate analysis revealed that lower histological or nuclear grade was also correlated with significantly longer OS (2 vs. $1 ; P=0.021)$ (3 vs. 1 ; $P<0.001$ ) (Table 3).

\section{Analysis of recurrent metastatic cases}

We analyzed the correlation between DFI and OS in recurrent metastatic cases $(n=824)$. By univariate analysis (Table 4 ), younger age ( $<60$ years), earlier treatment line of fulvestrant, longer time from $\mathrm{ABC}$ diagnosis to fulvestrant use ( $\geq 3$ years), no prior palliative chemotherapy, and lower histological or nuclear grade were associated with longer OS $(P<0.1)$. By multivariate analysis, younger age ( $<60$ years; $P<0.0001$ ), longer time from ABC diagnosis to fulvestrant use ( $\geq 3$ years; $P<0.001$ ), and no prior

Table 2 Univariate and multivariate Cox proportional hazards regression models for OS: all patients $(n=1031)$

\begin{tabular}{|c|c|c|c|c|c|c|}
\hline \multirow[t]{2}{*}{ Characteristic } & \multicolumn{3}{|c|}{ Univariate analysis } & \multicolumn{3}{|c|}{ Multivariate analysis ${ }^{\mathrm{a}}$} \\
\hline & HR & $95 \% \mathrm{CI}$ & $p$ value & HR & $95 \% \mathrm{CI}$ & $P$ value \\
\hline Age $(\mathrm{ABC}$ diagnosis $)(\geq 60$ years vs. $<60)$ & 1.59 & $1.36-1.85$ & $<0.001$ & 1.35 & $1.15-1.58$ & $<0.001$ \\
\hline $\begin{array}{l}\text { Treatment line of fulvestrant (4th or more vs. 3rd vs. 1st } \\
\text { and } 2 \text { nd) }\end{array}$ & 0.68 & $0.61-0.75$ & $<0.001$ & 1.00 & $0.90-1.12$ & 0.965 \\
\hline ABC diagnosis to fulvestrant use ( $\geq 3$ years vs. $<3$ ) & 0.16 & $0.13-0.19$ & $<0.001$ & 0.14 & $0.12-0.18$ & $<0.001$ \\
\hline Prior palliative chemotherapy (yes vs. no) & 0.78 & $0.67-0.90$ & 0.001 & 1.44 & $1.21-1.71$ & $<0.001$ \\
\hline \multicolumn{7}{|l|}{ Histological or nuclear grade } \\
\hline (2 vs. 1$)$ & 1.74 & $1.45-2.08$ & $<0.001$ & & & \\
\hline (3 vs. 1$)$ & 2.30 & $1.86-2.85$ & $<0.001$ & & & \\
\hline Visceral metastasis (yes vs. no) & 0.97 & $0.83-1.13$ & 0.685 & & & \\
\hline PgR (positive vs. negative) & 1.17 & $0.98-1.39$ & 0.089 & 1.29 & $1.06-1.59$ & 0.013 \\
\hline HER2 (positive vs. negative) & 0.84 & $0.65-1.09$ & 0.200 & & & \\
\hline
\end{tabular}

$O S$ overall survival, $H R$ hazard ratio, $C I$ confidence interval, $A B C$ advanced breast cancer, $P g R$ progesterone receptor HER2, human epidermal growth factor receptor 2

${ }^{\mathrm{a}} n=1030$ in the multivariate analysis (one patient was excluded because date of recurrence was unknown. Histological/nuclear grade data were not included in this analysis because one-third of the data were missing 
Table 3 Multivariate Cox proportional hazards regression models for OS using a dataset of all cases had grade information $(n=679)$

\begin{tabular}{lllr}
\hline Characteristic & HR & $95 \%$ CI & $p$ value \\
\hline Age (ABC diagnosis), ( $\geq 60$ vs. $<60$ years) & 1.16 & $0.96-1.40$ & 0.130 \\
Treatment line of fulvestrant (4th or more vs. 3rd vs. 1st and & 1.02 & $0.90-1.16$ & 0.754 \\
2nd) & & & \\
ABC diagnosis to fulvestrant use ( $\geq 3$ vs. $<3$ years) & 0.18 & $0.14-0.24$ & $<0.001$ \\
Prior palliative chemotherapy (yes vs. no) & 1.37 & $1.12-1.67$ & 0.002 \\
PgR (positive vs. negative) & 1.20 & $0.95-1.53$ & 0.131 \\
Histological or nuclear grade & & & \\
(2 vs. 1) & 1.28 & $1.04-1.59$ & 0.021 \\
(3 vs. 1) & 1.78 & $1.39-2.28$ & $<0.001$ \\
\hline
\end{tabular}

$O S$ overall survival, $H R$ hazard ratio, $C I$ confidence interval, $A B C$ advanced breast cancer, PgR, progesterone receptor

Table 4 Univariate and multivariate Cox proportional hazards regression models for OS using a dataset of recurrent metastatic cases $(n=824)$

\begin{tabular}{|c|c|c|c|c|c|c|}
\hline \multirow[t]{2}{*}{ Characteristic } & \multicolumn{3}{|c|}{ Univariate analysis } & \multicolumn{3}{|c|}{ Multivariate analysis $^{\mathrm{a}}$} \\
\hline & HR & $95 \% \mathrm{CI}$ & $p$ value & HR & $95 \% \mathrm{CI}$ & $p$ value \\
\hline Age (ABC diagnosis), ( $\geq 60$ vs. $<60$ years) & 1.66 & $1.40-1.98$ & $<0.001$ & 1.42 & $1.19-1.70$ & $<0.001$ \\
\hline $\begin{array}{l}\text { Treatment line of fulvestrant (4th or more vs. 3rd vs. 1st } \\
\text { and 2nd) }\end{array}$ & 0.70 & $0.62-0.78$ & $<0.001$ & 1.00 & $0.89-1.13$ & 0.992 \\
\hline $\mathrm{ABC}$ diagnosis to fulvestrant use ( $\geq 3$ vs. $<3$ years) & 0.16 & $0.13-0.19$ & $<0.001$ & 0.14 & $0.11-0.18$ & $<0.001$ \\
\hline Prior palliative chemotherapy (yes vs. no) & 0.78 & $0.66-0.93$ & 0.004 & 1.45 & $1.20-1.76$ & $<0.001$ \\
\hline \multicolumn{7}{|l|}{ Histological or nuclear grade } \\
\hline (2 vs. 1$)$ & 1.73 & $1.41-2.12$ & $<0.001$ & & & \\
\hline (3 vs. 1 ) & 2.58 & $2.03-3.27$ & $<0.001$ & & & \\
\hline Visceral metastasis (yes vs. no) & 0.99 & $0.83-1.17$ & 0.898 & & & \\
\hline PgR (positive vs. negative) & 1.14 & $0.94-1.38$ & 0.200 & & & \\
\hline HER2 (positive vs. negative) & 0.87 & $0.65-1.17$ & 0.360 & & & \\
\hline DFI ( $\geq 5$ vs. $<5$ years $)$ & 1.00 & $0.84-1.18$ & 0.950 & & & \\
\hline
\end{tabular}

$O S$ overall survival, $H R$ hazard ratio, $C I$ confidence interval, $A B C$ advanced breast cancer, $P g R$ progesterone receptor HER2, human epidermal growth factor receptor 2, DFI disease-free interval

${ }^{\text {a }} n=823$ in the multivariate analysis (one patient was excluded because the date of recurrence was unknown. Histological/nuclear grade data were not included in this analysis because one-third of the data were missing

palliative chemotherapy $(P<0.001)$ were associated with significantly longer OS (Table 4). DFI was not a factor related to OS. Histological or nuclear grade were not included in this multivariate analysis because data were missing in one-third of cases.

\section{Analysis of recurrent metastatic cases with available histological or nuclear grade data}

Multivariate analysis for OS in recurrent metastatic cases for which histological or nuclear grades were known showed that lower histological or nuclear grade also correlated with significantly longer OS (3 vs. $1 ; P<0.001)$ (Table 5).

\section{Discussion}

This study was an OS analysis of the large-scale, retrospective Safari study, a cohort study that analyzed the clinical outcomes of fulvestrant-treated $\mathrm{ER}+\mathrm{ABC}$ patients in Japan $[15,16]$. The median OS in ER+ patients was 7.0 years in our study, which was longer than the OS from similar cohort studies (3-5 years) [21-23]. One reason may be that the database of the Safari study included all cases of fulvestrant administration, including good-prognosis cases receiving fulvestrant after long-term endocrine therapy. Another reason may be the so-called lead-time bias, because physicians usually test tumor markers for follow-up of breast cancer patients after surgery in Japan 
Table 5 Multivariate Cox proportional hazards regression models for TTF using a dataset of recurrent metastatic cases that had grade information $(n=549)$

\begin{tabular}{lccr}
\hline Characteristic & HR & $95 \%$ CI & $p$ value \\
\hline Age (ABC diagnosis), ( $\geq 60$ vs. $<60$ years) & 1.27 & $1.03-1.57$ & 0.026 \\
Treatment line of fulvestrant & 1.00 & $0.87-1.14$ & 0.964 \\
(4th or more vs. 3rd vs. 1st and 2nd) & & & \\
ABC diagnosis to fulvestrant use ( $\geq 3$ vs. $<3$ years) & 0.18 & $0.13-0.24$ & $<0.001$ \\
Prior palliative chemotherapy use (yes vs. no) & 1.41 & $1.12-1.77$ & 0.003 \\
Histological or nuclear grade & & & \\
(2 vs. 1) & 1.21 & $0.95-1.53$ & 0.122 \\
(3 vs. 1) & 1.95 & $1.48-2.57$ & $<0.001$ \\
\hline
\end{tabular}

$O S$ overall survival, $H R$ hazard ratio, $C I$ confidence interval, $A B C$ advanced breast cancer, $P g R$ progesterone receptor HER2

${ }^{\mathrm{a}}$ Histological or nuclear grade data are included $(n=558)$
[24]. "Lead time" is the length of time between the detection of a disease and its usual clinical presentation and diagnosis. By screening, the intention is to diagnose a disease earlier than it would be without screening. Without screening, the disease may be discovered later, when symptoms appear [25]. Another possibility is that Japan has a universal health insurance system, and anyone can receive insurance coverage $[26,27]$. Therefore, there are few cases of patients discontinuing treatment because of cost, and it can be considered that continuing treatment longer than overseas cases is another reason for longer OS [26, 27].

A critical purpose of this subgroup analysis was to examine the data from the Safari study with a focus on the potential effect that patient- or disease-related factors may have on $\mathrm{OS}$ for $\mathrm{ER}+\mathrm{ABC}$. Identifying predictive factors of poor prognosis for $\mathrm{ABC}$ is very important for decision-making in patient treatment. In this study, multivariate analysis showed that younger age $[22,28]$ and low histological or nuclear grade [29] correlated with longer OS, as previously reported. Age is a patient-related factor, while histological or nuclear grade is disease-related [19]. Moreover, no prior palliative chemotherapy was also associated with significantly longer OS. Physicians are considered to have the tendency to administer chemotherapy at an earlier line for cases judged to be endocrine resistant and that have a poor prognosis. Our study suggested that in clinical practice the former is correct.

One interesting finding from this study is the association between a longer period from $\mathrm{ABC}$ diagnosis to fulvestrant use and longer OS. This finding may indicate that patients who respond well to other prior therapies will also respond to fulvestrant, implying that a durable response to the previous drug correlates with a durable response to fulvestrant. The above four factors other than age, lower histological or nuclear grade, longer time from ABC diagnosis to fulvestrant use, and no prior palliative chemotherapy, were also factors with longer TTF of fulvestrant $[15,16]$. Because the fulvestrant treatment line varied in this study, the fulvestrant
TTF length and OS length of ER+ ABC patients cannot simply be compared, but the fulvestrant TTF length may be a surrogate marker for subsequent OS.

Previous studies have also demonstrated that longer DFI $[10-12,22]$ and no visceral metastasis $[10-13,22]$ were positively correlated with longer OS in ABC. However, these factors were not associated with OS in our multivariate analysis. In this cohort, the cases with common poor prognostic factors, including short DFI and visceral metastases, might have had endocrine therapy discontinued prior to fulvestrant use, which was mostly used as a second- or later-line endocrine therapy, and shifted to chemotherapy without being treated with fulvestrant. Consequently, these patients were not included in the analyzed cohort, which may be the reason why, in this cohort, multivariate analysis did not extract these prognostic factors. Regarding the DFI cut-off, described in our previous report about TTF, we used "DFI ( $\geq 2$ vs. $<2$ years)". We used a cut-off of 2 years, as explained in the ESO-ESMO guidelines about intrinsic or primary endocrine resistance for early breast cancer, if defined as recurrence within the first 2 years of adjuvant endocrine therapy [4]. Nonetheless, in this analysis, we wanted to examine factors related to OS rather than endocrine therapy sensitivity, so the cut-off was set at a median of 5 years instead of 2 years. In fact, we also performed an analysis with a cut-off of 2 years, but the multivariate analysis did not show any correlation between DFI and OS (data not shown).

One confusing result of our study is that PgR- correlated with longer OS by multivariate analysis, which is the opposite to previous reports $[10,11]$. This result is difficult to explain logically, however, probably due to the selection bias of this study or confounding factors not defined in our analysis.

In recent years, molecular targeting drugs such as CDK 4/6 inhibitors and mTOR inhibitor have been administered in ER+ HER2 - ABC in combination with endocrine therapy, and endocrine therapy plus molecular targeted 
therapy is administered as an early-line treatment for ER+ HER2- ABC. At present, it is not possible to obtain data on the sequential administration of hormonal monotherapy as in the Safari study, so the significance of creating this database is profound. Even in the era of molecular targeting therapy combinations, continuation of molecular targeting drugs after tumor progression, so-called "beyond progression", is not recommended [30], and analysis using data of sequential administration of hormonal therapy that is the basis of ER+ HER2- breast cancer treatment is important. In the future, we plan to conduct research to analyze the effect of treatment order on OS by dynamic treatment regimen analysis [31] using the database from the Safari study.

As with the original Safari study, the limitations of our analysis include its retrospective design and the absence of a comparative treatment group. Moreover, there is a high bias due to selection of the fulvestrant-treated cohort, which probably does not reflect the ER+ ABC population. In addition, our results are considered to reflect real clinical practice. We initiated this study to examine the factors in the main analysis that affect fulvestrant TTF. Therefore, the OS analysis also included the treatment line of fulvestrant, the period from $\mathrm{ABC}$ diagnosis to fulvestrant use, and palliative chemotherapy prior to fulvestrant administration. We did not consider other endocrine therapies, and thus this is also a limitation of this study.

In conclusion, in $\mathrm{ER}+\mathrm{ABC}$ patients who received fulvestrant, younger age, longer period from recurrent breast cancer diagnosis to fulvestrant use, and low histological or nuclear grade, were associated with longer OS. These factors may be the key to predicting poor prognosis in patients with $\mathrm{ER}+\mathrm{ABC}$ whose treatment history includes fulvestrant.

Acknowledgements We thank the patients who participated in this study. We appreciate the contribution of data entry assistance from AC Medical INC, and the contribution of the Japan Breast Cancer Research Group (JBCRG) administrative office and IBEC Co., Ltd. to the management of the study. We thank Libby Cone, MD, MA, and $\mathrm{H}$. Nikki March, PhD, from Edanz Group Japan (www.edanzediting.com/ ac) for editing a draft of this manuscript.

Funding Funding for this study was provided by the Japan Breast Cancer Research Group (JBCRG), a study group that also participated in the conduct of the study, and AstraZeneca.

\section{Compliance with ethical standards}

Conflict of interest H. Kawaguchi received personal fees from Pfizer, Chugai, AstraZeneca, Eli Lilly, Eisai, Kyowa Kirin, Novartis, Taiho. Takeda. N. Masuda received grants, personal fees, and other from Chugai and Eisai, personal fees and other from AstraZeneca, Pfizer, Eli-Lilly, Takeda, Kyowa-Kirin, Novartis, and Daiichi Sankyo, and other from MSD. T. Nakayama received personal fees from AstraZeneca, Chugai, Novartis, Eli Lilly, Pfizer, Takeda. Taiho. K. Aogi received grants and personal fees from Eisai, personal fees from AstraZeneca, Daiichi Sankyo, Chugai, Taiho, Eli Lilly, Mochida, and Ono. K. Anan received personal fees from Pfizer, Chugai, AstraZeneca, Eisai and Novartis. Y. Ito received grants from Daiichi Sankyo, Chugai, Novartis, Parexel, EPS, MSD, AstraZeneca, Eli Lilly, Kyowa Hakko Kirin, Covance, Taiho, A2 Healthcare, QVIA services Japan, and Esai. S. Ohtani received personal fees from AstraZeneca, Pfizer, and Chugai. N. Sato received personal fees from AstraZeneca, Chugai, Eisai, and Taiho. S. Saji received grants and personal fees from Eisai, Chugai, AstraZeneca, Takeda, Novartis, Taiho, and Nihon Kayaku, personal fees from Kyowa Hakko Kirin, Pfizer, and Daiichi Sankyo, and grants from Ono. T. Takano received grants and personal fees from Daiichi Sankyo, Kyowa Hakko Kirin, and Eisai, grants from Ono, MSD, Merck Serono, Taiho, Novartis, and Chugai, and personal fees from Pfizer and Eli Lilly. E. Tokunaga received personal fees from Astra Zeneca and Pfizer. S. Nakamura received grants and personal fees from Novartis, Taiho and Takeda, grants from Pfizer; and personal fees from Nippon Kayaku and Shionogi. S. Morita received personal fees from Astra Zeneca. M. Yamaguchi received personal fees from Eli Lilly, Pfizer, Chugai Astra Zeneca, and Eisai. H. Yamashita received personal fees from Astra Zeneca, Takeda, Pfizer, Chugai Hitachi, Taiho, Daiichi Sankyo, Kyowa Hakko Kirin, Eli Lilly, Novartis, Nippon Kayaku, and Eisai. T. Yamashita received grants and other from Chugai, Nippon Kayaku, and Kyowa Hakko Kirin, and other from Eisai, Novartis Pharma, Taiho, AstraZeneca, and Pfizer. Dr. Yamamoto received personal fees from AstraZeneca, Pfizer, Nippon Kayaku, Kyowa Hakko Kirin, Chugai, Esai, Eli Lilly, Takeda, Sysmex, GE Health Care, and Daiich Sankyo. M. Toi received grants and personal fees from Taiho, Chugai, Takeda, Shimadzu, Eisai, Daiichi Sankyo, Pfizer, AstraZeneca, C\&C Res Lab, and Kyowa-Hakko-Kirin, personal fees from Yakult, Konica-Minolta, Eli Lilly, MSD, Genomic Health, Novartis, Nihon Kayaku, and Ono, grants and non-financial support from Jpn Breast Cancer Research Group association, and non-financial support from Jpn Society Clinical Oncology, Jpn Surgical Society, Jpn Breast Cancer Society, Jpn Breast Cancer Research Group organization, Organisation for Oncology and Translational Research, and Kyoto Breast Cancer Research Network. S. Ohno received grants and personal fees from Eisai and Taiho, and personal fees from AstraZeneca, Chugai, Pfizer, Novartis, Nippon Kayaku, Kyowa Hakko Kirin, and Eli Lilly. Y. Hasegawa, Masaya Hattori, T. Fujisawa, and D. Yotsumoto have no financial or other relationships to declare.

Open Access This article is licensed under a Creative Commons Attribution 4.0 International License, which permits use, sharing, adaptation, distribution and reproduction in any medium or format, as long as you give appropriate credit to the original author(s) and the source, provide a link to the Creative Commons licence, and indicate if changes were made. The images or other third party material in this article are included in the article's Creative Commons licence, unless indicated otherwise in a credit line to the material. If material is not included in the article's Creative Commons licence and your intended use is not permitted by statutory regulation or exceeds the permitted use, you will need to obtain permission directly from the copyright holder. To view a copy of this licence, visit http://creativecommons.org/licenses/by/4.0/.

\section{References}

1. EBCTCG (Early Breast Cancer Trialists' Collaborative Group), McGale P, Taylor C, Correa C, Cutter D, Duane F, et al. Effect of radiotherapy after mastectomy and axillary surgery on 10-year recurrence and 20-year breast cancer mortality: meta-analysis of individual patient data for 8135 women in 22 randomised trials. Lancet. 2014;383:2127-35. https://doi.org/10.1016/S0140 $-6736(14) 60488-8$.

2. Redig AJ, McAllister SS. Breast cancer as a systemic disease: a view of metastasis. J Intern Med. 2013;274:113-26. https://doi. org/10.1111/joim.12084. 
3. Cardoso F, Harbeck N, Fallowfield L, Kyriakides S, Senkus E. Locally recurrent or metastatic breast cancer: ESMO clinical practice guidelines for diagnosis, treatment and follow-up. Ann Oncol. 2012;23:vii11-9. https://doi.org/10.1093/annonc/mds232.

4. Cardoso F, Costa A, Senkus E, Aapro M, André F, Barrios CH, et al. 3rd ESO-ESMO International consensus guidelines for Advanced Breast Cancer (ABC 3). Breast. 2017;31:244-59. https ://doi.org/10.1016/j.breast.2016.10.001.

5. Migliaccio I, Malorni L, Hart CD, Guarducci C, Di Leo A. Endocrine therapy considerations in postmenopausal patients with hormone receptor positive, human epidermal growth factor receptor type 2 negative advanced breast cancers. BMC Med. 2015;13:46. https://doi.org/10.1186/s12916-015-0280-0.

6. Başaran GA, Twelves C, Diéras V, Cortés J, Awada A. Ongoing unmet needs in treating estrogen receptor positive/HER2-negative metastatic breast cancer. Cancer Treat Rev. 2018;63:144-55. https ://doi.org/10.1016/j.ctrv.2017.12.002.

7. Brodowicz T, Lang I, Kahan Z, Greil R, Beslija S, Stemmer SM, et al. Selecting first-line bevacizumab-containing therapy for advanced breast cancer: TURANDOT risk factor analyses. Br J Cancer. 2014;111:2051-7. https://doi.org/10.1038/bjc.2014.504.

8. Zhang XH, Giuliano M, Trivedi MV, Schiff R, Osborne CK. Metastasis dormancy in estrogen receptor-positive breast cancer. Clin Cancer Res. 2013;19:6389-97. https://doi.org/10.1158/10780432.CCR-13-0838.

9. Ignatiadis M, Sotiriou C. Luminal breast cancer: from biology to treatment. Nat Rev Clin Oncol. 2013;10:494-506. https://doi. org/10.1038/nrclinonc.2013.124.

10. Llombart-Cussac A, Pivot X, Biganzoli L, Cortes-Funes H, Pritchard KI, Pierga JY, et al. A prognostic factor index for overall survival in patients receiving first-line chemotherapy for HER2negative advanced breast cancer: an analysis of the ATHENA trial. Breast. 2014;23:656-62. https://doi.org/10.1016/j.breas t.2014.06.017.

11. Sledge GW, Neuberg D, Bernardo P, Ingle JN, Martino S, Rowinsky EK, et al. Phase III trial of doxorubicin, paclitaxel, and the combination of doxorubicin and paclitaxel as front-line chemotherapy for metastatic breast cancer: an intergroup trial (E1193). J Clin Oncol. 2003;21:588-92. https://doi.org/10.1200/ JCO.2003.08.013.

12. Yamamoto N, Watanabe T, Katsumata N, Omuro Y, Ando $\mathrm{M}$, Fukuda $\mathrm{H}$, et al. Construction and validation of a practical prognostic index for patients with metastatic breast cancer. J Clin Oncol. 1998;16:2401-8. https://doi.org/10.1200/ JCO.1998.16.7.2401.

13. Yamamura J, Kamigaki S, Tsujie M, Fujita J, Osato H, Higashi $\mathrm{C}$, et al. Response to first-line recurrence treatment influences survival in hormone receptor-positive, HER2-negative breast cancer: a multicenter study. Vivo. 2019;33:281-7. https://doi. org/10.21873/invivo.11473.

14. Ogiya A, Yamazaki K, Horii R, Shien T, Horimoto Y, Masuda N, et al. Collaborative Study Group of Scientific Research of the Japanese Breast Cancer Society. Post-relapse survival in patients with the early and late distant recurrence in estrogen receptor positive HER2-negative breast cancer. Breast Cancer 2017;24:473-82. https://hdl.handle.net/2115/70047

15. Kawaguchi H, Masuda N, Nakayama T, Aogi K, Anan K, Ito $\mathrm{Y}$, et al. Outcomes of fulvestrant therapy among Japanese women with advanced breast cancer: a retrospective multicenter cohort study (JBCRG-C06; Safari). Breast Cancer Res Treat. 2017;163:545-54. https://doi.org/10.1007/s10549-017-4212-x.

16. Kawaguchi H, Masuda N, Nakayama T, Aogi K, Anan K, Ito Y, et al. Factors associated with prolonged time to treatment failure with fulvestrant $500 \mathrm{mg}$ in patients with post-menopausal estrogen receptor-positive advanced breast cancer: a sub-group analysis of the JBCRG-C06 Safari study. Curr Med Res Opin.
2018;34:49-544. https://doi.org/10.1080/03007995.2017.14004 26.

17. Faslodex ${ }^{\circledR}$ summary of product characteristics. London (UK): European Medicines Agency; 2019. https://www.ema.europa.eu/ en/documents/product-information/faslodex-epar-product-infor mation_en.pdf. Accessed 21 May 2019

18. Faslodex ${ }^{\circledR}$ prescribing information. Silver Spring (MD): US Food and Drug Administration; 2019. https://www.accessdata.fda.gov/ drugsatfda_docs/label/2019/021344s037lbl.pdf. Accessed 21 May 2019

19. Cardoso F, Harbeck N, Fallowfield L, Kyriakides S, Senkus E, ESMO Guidelines Working Group. Locally recurrent or metastatic breast cancer: ESMO Clinical Practice Guidelines for diagnosis, treatment and follow-up. Ann Oncol. 2012;23(Suppl 7):vii11-9.

20. National Comprehensive Cancer Network. Invasive breast cancer (Version 4.2018). Fort Washington (PA): NCCN; 2017. https ://www.nccn.org/professionals/physician_gls/f_guidelines.asp. Accessed 5 May 2019

21. Caswell-Jin JL, Plevritis SK, Tian L, Cadham CJ, Xu C, Stout NK, et al. Change in survival in metastatic breast cancer with treatment advances: meta-analysis and systematic review. JNCI Cancer Spectr. 2018;2:pky062. https://doi.org/10.1093/jncics/pky062.

22. Lobbezoo DJ, van Kampen RJ, Voogd AC, Dercksen MW, van den Berkmortel F, Smilde TJ, et al. Prognosis of metastatic breast cancer: are there differences between patients with de novo and recurrent metastatic breast cancer? Br J Cancer. 2015;112:1445511. https://doi.org/10.1038/bjc.2015.127.

23. Chen MT, Sun HF, Zhao Y, Fu WY, Yang LP, Gao SP, et al. Comparison of patterns and prognosis among distant metastatic breast cancer patients by age groups: a SEER population-based analysis. Sci Rep. 2017;23(7):9254. https://doi.org/10.1038/s4159 8-017-10166-8.

24. Hojo T, Masuda N, Mizutani T, Shibata T, Kinoshita T, Tamura $\mathrm{K}$, et al. Intensive vs. standard post-operative surveillance in highrisk breast cancer patients (INSPIRE): Japan Clinical Oncology Group Study JCOG1204. Jpn J Clin Oncol. 2015;45:983-6. https ://doi.org/10.1093/jjco/hyv110.

25. Gordis L. Epidemiology. Philadelphia: Saunders; 2008. p. 318.

26. Abe S. Japan's vision for a peaceful and healthier world. Lancet. 2015;386:2367-9. https://doi.org/10.1016/S0140-6736(15)01172 $-1$.

27. Abe S. Japan's strategy for global health diplomacy: why it matters. Lancet. 2013;382:915-6. https://doi.org/10.1016/S0140 $-6736(13) 61639-6$.

28. Largillier R, Ferrero JM, Doyen J, Barriere J, Namer M, Mari V, et al. Prognostic factors in 1,038 women with metastatic breast cancer. Ann Oncol. 2008;19:2012-9. https://doi.org/10.1093/ annonc/mdn424.

29. Sánchez C, Camus M, Medina L, Oddo D, Artigas RC, Pérez Sepúlveda A, et al. Clinico-pathologic subtypes of breast cancer primary tumors are related to prognosis after recurrence. Asian Pac J Cancer Prev. 2016;17:5081-6.

30. Cardoso F, Senkus E, Costa A, Papadopoulos E, Aapro M, André $\mathrm{F}$, et al. 4th ESO-ESMO international consensus guidelines for advanced breast cancer (ABC 4). Ann Oncol. 2018;29:1634-57. https://doi.org/10.1093/annonc/mdy192.

31. Huang X, Cormier JN, Pisters PW. Estimation of the causal effects on survival of two-stage nonrandomized treatment sequences for recurrent diseases. Biometrics. 2006;62:901-9.

Publisher's Note Springer Nature remains neutral with regard to jurisdictional claims in published maps and institutional affiliations. 


\section{Affiliations}

Hidetoshi Kawaguchi ${ }^{1}$ (D) Norikazu Masuda ${ }^{2}$ - Takahiro Nakayama ${ }^{3} \cdot$ Kenjiro Aogi $^{4} \cdot$ Keisei Anan $^{5} \cdot$ Yoshinori Ito $^{6}$. Shoichiro Ohtani ${ }^{7}$. Nobuaki Sato ${ }^{8}$. Shigehira Saji ${ }^{9} \cdot$ Toshimi Takano $^{10}$. Eriko Tokunaga ${ }^{11}$. Seigo Nakamura ${ }^{12}$. Yoshie Hasegawa ${ }^{13} \cdot$ Masaya Hattori $^{14} \cdot$ Tomomi Fujisawa $^{15}$. Satoshi Morita ${ }^{16} \cdot$ Miki Yamaguchi $^{17}$. Hiroko Yamashita ${ }^{18}$. Toshinari Yamashita ${ }^{19}$. Yutaka Yamamoto ${ }^{20}$. Daisuke Yotsumoto ${ }^{21}$. Masakazu Toi ${ }^{22}$. Shinji Ohno ${ }^{23}$

1 Department of Breast Surgery, Matsuyama Red Cross Hospital, 1 Bunkyo-cho, Matsuyama 790-8524, Japan

2 Department of Surgery, Breast Oncology, NHO Osaka National Hospital, Osaka 540-0006, Japan

3 Department of Breast and Endocrine Surgery, Osaka International Cancer Institute, Osaka 541-8567, Japan

4 Department of Breast Oncology, NHO Shikoku Cancer Center, Matsuyama 791-0280, Japan

5 Department of Surgery, Kitakyushu Municipal Medical Center, Kitakyushu 802-0077, Japan

6 Department of Breast Medical Oncology, The Cancer Institute Hospital of JFCR, Tokyo 135-8550, Japan

7 Department of Breast Surgery, Hiroshima City Hiroshima Citizens Hospital, Hiroshima 730-8518, Japan

8 Department of Breast Oncology, Niigata Cancer Center Hospital, Niigata 951-8566, Japan

9 Department of Medical Oncology, Fukushima Medical University, Fukushima 960-1295, Japan

10 Department of Medical Oncology, Toranomon Hospital, Tokyo 105-8470, Japan

11 Department of Breast Oncology, Kyushu Cancer Center, Fukuoka 811-1395, Japan

12 Department of Surgery, Division of Breast Surgical Oncology, Showa University School of Medicine, Tokyo 142-8666, Japan
13 Department of Breast Surgery, Hirosaki Municipal Hospital, Hirosaki 036-8004, Japan

14 Department of Breast Oncology, Aichi Cancer Center Hospital, Nagoya 464-8681, Japan

15 Department of Breast Oncology, Gunma Prefectural Cancer Center, Ohta 373-8550, Japan

16 Department of Biomedical Statistics and Bioinformatics, Graduate School of Medicine, Kyoto University, Kyoto 606-8507, Japan

17 Department of Breast Surgery, JCHO Kurume General Hospital, Kurume 830-0013, Japan

18 Department of Breast Surgery, Hokkaido University Hospital, Sapporo 060-8648, Japan

19 Department of Breast and Endocrine Surgery, Kanagawa Cancer Center, Yokohama 241-8515, Japan

20 Department of Breast and Endocrine Surgery, Kumamoto University Graduate School of Medical Sciences, Kumamoto 860-8556, Japan

21 Department of Breast Surgical Oncology, Hakuaikai Medical Corporation Sagara Hospital, Kagoshima 892-0833, Japan

22 Department of Breast Surgery, Graduate School of Medicine, Kyoto University, Kyoto 606-8507, Japan

23 Breast Oncology Center, The Cancer Institute Hospital of JFCR, Tokyo 135-8550, Japan 